\title{
Controle químico de Talinum paniculatum em três estádios de desenvolvimento ${ }^{1}$
}

\section{Chemical control of Talinum paniculatum in three stages of development}

\author{
André Prechlak Barbosa ${ }^{2}$; André Felipe Moreira Silva ${ }^{3}$; Alfredo Júnior Paiola Albrecht ${ }^{4}$; Arthur \\ Arrobas Martins Barroso ${ }^{5}$; Ricardo Victória Filho ${ }^{6}$; Leandro Paiola Albrecht ${ }^{4}$; Victor Volante \\ Garcia $^{7}$
}

Resumo - Talinum paniculatum é uma espécie pertencente à família Portulacacea, conhecida popularmente como maria-gorda, planta daninha em culturas como a cana-de-açúcar, feijão comum, soja, milho e cenoura, além de áreas não agrícolas. Com este trabalho objetivou-se estudar a eficácia de herbicidas no controle de $T$. paniculatum, assim como seu efeito na massa fresca e seca destas plantas em três estádios de desenvolvimento. $\mathrm{O}$ delineamento experimental utilizado foi o inteiramente casualizado, com nove tratamentos e quatro repetições, posicionados em três estádios: 4-6 folhas, 10-12 folhas e florescimento. Para avaliar a eficácia dos tratamentos aferiu-se a porcentagem de controle das plantas, além de sua massa fresca e seca. Houve maior controle para alguns tratamentos dentro das fases de desenvolvimento das plantas daninhas, com aplicação de isoxaflutole, diuron+hexazinone, ametryn e imazapic no primeiro estádio, glyphosate na dose de 2000 g e.a. ha ${ }^{-1}$, diuron+hexazinone e 2,4-D no segundo estádio e 2,4-D, glyphosate na dose de $2000 \mathrm{~g}$ e.a. ha ${ }^{-1}$ e amônio-glufosinato no terceiro estádio. A massa fresca e seca das plantas daninhas foi afetada, seguindo o comportamento obtido para o controle. $\mathrm{O}$ uso de herbicidas se mostrou eficiente na minoria dos arranjos testados para o controle de T. paniculatum. $\mathrm{O}$ estádio de desenvolvimento da planta influencia a eficácia de controle dos tratamentos.

Palavras-chaves: estádio fenológico, Portulacaceae, herbicidas

Abstract - Talinum paniculatum is a species of the Portulacacea family, popularly known as "maria-gorda", weed in crops such as sugarcane, common bean, soybean, corn and carrot, as well as in non-agricultural areas. This work aimed to study the efficacy of herbicides in the control of

\footnotetext{
${ }^{1}$ Recebido para publicação em 11/02/2015 e aceito em 09/07/2015.

${ }^{2}$ Mestrando em Agronomia, Universidade Estadual de Londrina (UEL), Centro de Ciências Agrárias. Rodovia Celso Garcia Cid Km 380, CEP: 86057-970, Campus Universitário, Londrina, PR. E-mail: <andreprechlak@gmail.com>. ${ }^{3}$ Mestrando em Fitotecnia, Universidade de São Paulo - Escola Superior de Agrícola “Luiz de Queiroz" (USP/ESALQ) Rua Pádua Dias, nº11, CEP: 13418-900, Piracicaba, SP. E-mail: <afmoreirasilva@usp.br>.

${ }^{4}$ Docentes do curso de graduação em Agronomia, Universidade Federal do Paraná (UFPR), Setor Palotina, PR. Rua Pioneiro, $\mathrm{n}^{\mathrm{o}}$ 2153, Jardim Dallas, CEP 85950-000, Palotina, PR. E-mail: <ajpalbrecht@yahoo.com.br> e <lpalbrecht@yahoo.com.br>.

5 Doutorando em Agronomia, Universidade Estadual Paulista "Júlio de Mesquita Filho" (UNESP), Faculdade de Ciências Agrárias e Veterinárias. Vila Industrial, CEP: 14884-12 900, Jaboticabal, SP. E-mail: <arthuragro07@hotmail.com>.

${ }^{6}$ Docente do curso de Engenharia Agronômica, Universidade de São Paulo - Escola Superior de Agrícola "Luiz de Queiroz” (USP/ESALQ) Rua Pádua Dias, n¹1, CEP: 13418-900, Piracicaba, SP. E-mail: rvictori@usp.br.

${ }^{7}$ Graduando em Agronomia, Universidade Estadual de Londrina (UEL), Centro de Ciências Agrárias. Rodovia Celso Garcia Cid Km 380, CEP: 86057-970, Campus Universitário, Londrina, PR. E-mail: <victor.volante@hotmail.com>.
} 
T. paniculatum, as well as its effect on fresh and dry mass of these plants in three stages of development. The experimental design was completely randomized, with nine treatments and four replications, positioned in three stages: 4-6 leaves, 10-12 leaves and flowering. To evaluate the effectiveness of the treatments, the percentage of the control of plants was evaluated, in addition to its fresh and dry mass. There was higher control for some treatments within the development stages of weeds, with application of isoxaflutole, diuron+hexazinone, ametryn, and imazapic in the first stage, glyphosate at the dose of $2000 \mathrm{~g}$ a.e. ha ${ }^{-1}$, diuron+hexazinone and 2,4-D in the second stage, and 2,4-D, glyphosate at $2000 \mathrm{~g}$ a.e. $\mathrm{ha}^{-1}$, and ammonium-glufosinate in the third stage. Fresh and dry mass of weeds were affected, following the behavior obtained for the evaluations of control. The use of herbicides was efficient in the minority of arrangements tested for control of $T$. paniculatum. The development stage of the plant was influenced by the efficacy control of treatments.

Keywords: phenological stage, Portulacaceae, herbicides

\section{Introdução}

A sustentabilidade do manejo químico para o controle de plantas daninhas é um aspecto relevante dentro do sistema de produção agrícola atual, para isso, é essencial adequá-lo às situações e ter a consciência que para a obtenção de altos níveis de produtividade e consequentemente de rentabilidade para o agricultor, é crucial evitar ao máximo a interferência das plantas daninhas na cultura. Talinum paniculatum é uma portulacacea, pertencente à classe Liliopsida (monocotiledôneas), tem sua origem no continente americano, conhecida popularmente como maria-gorda e/ou bredo. Nessa mesma família estão Portulaca oleraceae e Talinum triangulare, que também possuem importância como plantas infestantes de culturas, beiras de estradas e terrenos baldios (Carvalho, 2013).

Portanto, o controle químico é destaque atualmente para a maioria das culturas, devido sua praticidade e muitas vezes pelo menor custo. A variedade de produtos atualmente é muito grande, estima-se que existam 516 herbicidas dentro de 11 grupos, destes são registrados apenas amônio glufosinato e glyphosate para $T$. paniculatum (Rodrigues e Almeida, 2011).

As Portulacaceas vêm demonstrando importância nos sistemas de produção, sendo a maria-gorda uma das plantas de recente ocorrência. Relatos em várias culturas têm sido feitos, em cana-crua, (Kuva et al., 2008;
Oliveira e Freitas, 2008), na bananicultura (Gomes et al., 2010; Moura filho et al., 2015), soja transgênica (Pittelkow et al., 2009), milho (Nascimento et al., 2012), feijão comum (Borchartt et al., 2011) e até no ambiente urbano onde sua presença foi recorrente em gramados de Botucatu-SP (Maciel et al., 2010) e bairros próximos da zona rural de João Pessoa-PB (Machado filho et al., 2015).

Se destacou como uma das espécies de maior densidade populacional, com um índice de ocorrência de 12,6\%, evidenciando sua importância como infestante na cultura do feijão-caupi (Freitas et al., 2009). Em trabalho realizado com a cultura da cenoura, $T$. paniculatum apresentou a densidade de 28 plantas por $\mathrm{m}^{2} \operatorname{logo}$ após a emergência da cultura, o controle eficiente da espécie só foi possível após a realização do capina por duas vezes (Soares et al., 2010). A espécie apresentou índice de valor de importância de 1,5\% em dois sistemas de consorciação com a cultura do pinhão manso, o que salienta a variedade de ecossistemas em que está presente (Concenço et al., 2014).

Verificada a dificuldade de controle e ocorrência dessa espécie nos sistemas produtivos e os danos por ela causados, têm-se a necessidade de gerar informações referentes ao seu manejo, assim como um melhor posicionamento do controle químico e as opções de herbicidas e doses que ofereçam eficiência. 
Portanto o objetivo do presente trabalho foi avaliar a eficiência de diferentes herbicidas no controle de $T$. paniculatum em três estádios de desenvolvimento.

\section{Material e Métodos}

Os três experimentos foram conduzidos em casa de vegetação na Escola Superior de Agricultura "Luiz de Queiroz" - Universidade de São Paulo (ESALQ - USP), no município de Piracicaba, Estado de São Paulo.

As unidades experimentais foram constituídas de vasos com capacidade volumétrica de $5 \mathrm{dm}^{-3}$, preenchidos com amostras de um solo classificado como
LATOSSOLO VERMELHO-AMARELO Distrófico típico, com textura média (Embrapa, 2006). Neles foram implantadas seções de raízes de $T$. paniculatum, que foram mantidos em casa de vegetação para seu crescimento, posteriormente foram separados em três experimentos: dois com as plantas em dois estádios vegetativos distintos (4-6 folhas - 5-10 $\mathrm{cm}$ de altura e 10-12 folhas - 10-20 cm de altura), sendo experimento 1 e 2 respectivamente e um experimento quando as plantas estavam no estádio reprodutivo (10-12 folhas, com a presença de inflorescência - 20-30 $\mathrm{cm}$ de altura), considerado como experimento 3 (Figura 1), não havendo comparação estatística entre os estádios estudados.

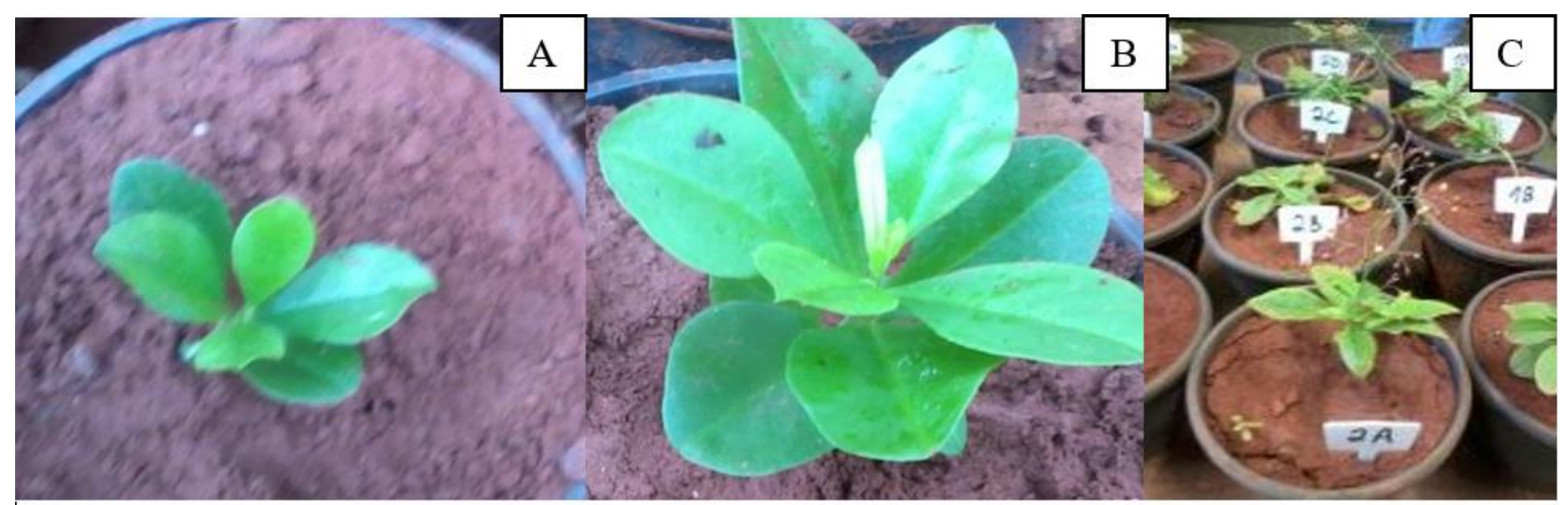

Figura 1. Plantas de Talinum paniculatum nos três estádios utilizados (A. 4-6 folhas, B. 10-12 folhas e C. com inflorescência). Piracicaba - SP, 2013.

O delineamento experimental utilizado foi o inteiramente casualizado, com nove tratamentos e quatro repetições, envolvendo os seguintes herbicidas: amônio glufosinato $(400 \mathrm{~g}$ i.a. ha $\left.{ }^{-1}\right)$, glyphosate $(1.000$ e 2.000 g e.a. ha-1), imazapic (140 g i.a. ha $\left.{ }^{-1}\right)$, ametryn $(2.500 \mathrm{~g}$ i.a. $\left.\mathrm{ha}^{-1}\right)$, diuron + hexazinone $(1170+330 \mathrm{~g}$ i.a. ha $\left.{ }^{1}\right), 2,4-\mathrm{D}$ (1.005 g i.a. ha ${ }^{-1}$ ), isoxaflutole (94 g i.a. $\left.\mathrm{ha}^{-1}\right)$ e testemunha, sem aplicação de herbicida.

As aplicações foram realizadas em 23/08/2013 (experimento 1) e 13/09/2013 (experimento 2 e 3), utilizou-se uma câmara de pulverização automatizada, com pulverizador utilizando pressão de 30 psi, contendo uma ponta tipo leque, com jato plano, modelo XR 80.02 , calibrada para um volume de calda de
$200 \mathrm{~L} \mathrm{ha}^{-1}$ para todos os tratamentos contendo herbicidas. Após a aplicação, os vasos foram acondicionados na casa de vegetação, sem irrigação por 24 horas, para garantir a absorção dos herbicidas; após esse período, todos os vasos receberam uma lâmina d'água proporcional a uma precipitação de $10 \mathrm{~mm}$, sendo irrigados diariamente em taxa variável, mantendo solo em estado friável.

$\mathrm{O}$ controle da $T$. paniculatum foi avaliado aos 7, 14, 21 e 28 dias após aplicação (DAA), considerando 0\% como ausência de controle e $100 \%$ a morte das plantas (SBCPD, 1995). Os parâmetros utilizados para estabelecimento das notas foram: acúmulo de biomassa, inibição do crescimento, quantidade e 
uniformidade das injurias, capacidade de rebrota das plantas.

Foi aferida ainda a massa fresca e seca de todo material vegetal remanescente da parte aérea das plantas aos 28 DAA. O material foi coletado e a massa fresca foi determinada. Após a secagem em estufa com ventilação forçada por $72 \mathrm{~h}$ a $65^{\circ} \mathrm{C}$ foi mensurada a massa seca em balança analítica, com precisão de três casas decimais.

Os dados obtidos foram submetidos à análise de variância e as suas médias comparadas pelo teste Tukey, ao nível de 5\% de probabilidade.

\section{Resultados e Discussão}

Os dados submetidos à análise estatística demonstraram que houve diferenciação ao nível de $5 \%$ de significância para todas as características estudadas dentro dos três estádios de aplicação (experimentos 1, 2 e 3) (Tabela 1).

Para a aplicação no primeiro estádio de desenvolvimento (experimento 1), não houve controle satisfatório até 28 dias após aplicação (DAA) para a maioria dos tratamentos (Tabela 2), houve apenas controle considerado eficiente, de $85 \%$, para as plantas daninhas com o herbicida isoxaflutole, possivelmente pela característica da molécula de maior absorção pelo sistema radicular e por sementes, sendo essa absorção exclusivamente via raízes quando o mesmo passa para forma de metabólito diquetonitrila (DKN), que possui maior efetividade no controle das plantas daninhas (Marchiori Jr. et al., 2005). Demonstrando que a interrupção da síntese dos carotenos teve um efeito de supressão interessante nas plantas ainda jovens de $T$. paniculatum, não permitindo às mesmas retomar seu crescimento e desenvolvimento.

Tabela 1. Análise de variância das variáveis de porcentagem de controle aos 7 DAA, 14 DAA, 21 DAA e 28 DAA, massa fresca (MF) e massa seca (MS) de T. paniculatum em função dos diferentes tratamentos. Piracicaba - SP, 2013.

\begin{tabular}{|c|c|c|c|c|c|c|}
\hline \multirow{3}{*}{$\begin{array}{c}\text { Fonte de variação } \\
\text { (Tratamentos) }\end{array}$} & \multicolumn{6}{|c|}{ Estádio 1} \\
\hline & \multicolumn{4}{|c|}{$\%$ de controle } & \multirow{2}{*}{ MF } & \multirow{2}{*}{ MS } \\
\hline & $7 \mathrm{DAA}$ & 14 DAA & $21 \mathrm{DAA}$ & 28 DAA & & \\
\hline GL & 8 & 8 & 8 & 8 & 8 & 8 \\
\hline SQ & 15583,33 & 13890,74 & 18516,67 & 27207,41 & 154,86 & 1,96 \\
\hline QM & 1947,92 & 1736,34 & 2314,58 & 3400,93 & 19,36 & 0,25 \\
\hline $\mathrm{Fc}$ & $175,31^{*}$ & $153,08^{*}$ & $82,29^{*}$ & $175,95^{*}$ & $354,57^{*}$ & $103,80^{*}$ \\
\hline \multirow[t]{2}{*}{$\mathrm{CV}(\%)$} & 6,98 & 6,14 & 11,64 & 8,76 & 7,28 & 14,90 \\
\hline & \multicolumn{6}{|c|}{ Estádio 2} \\
\hline GL & 8 & 8 & 8 & 8 & 8 & 8 \\
\hline SQ & 20876,39 & 22726,39 & 26980,56 & 27775,00 & 79,29 & 0,62 \\
\hline QM & 2609,55 & 2840,80 & 3372,57 & 3471,88 & 9,91 & 0,08 \\
\hline $\mathrm{Fc}$ & $101,33^{*}$ & $67,71^{*}$ & $253,38^{*}$ & $260,84^{*}$ & $17,38^{*}$ & $8,18^{*}$ \\
\hline \multirow[t]{2}{*}{$\mathrm{CV}(\%)$} & 18,93 & 11,16 & 5,04 & 4,95 & 28,52 & 30,39 \\
\hline & \multicolumn{6}{|c|}{ Estádio 3} \\
\hline$\overline{G L}$ & 8 & 8 & 8 & 8 & 8 & 8 \\
\hline SQ & 33301,39 & 36093,06 & 35712,50 & 42247,22 & 40,95 & 0,27 \\
\hline QM & 4162,67 & 4511,63 & 4464,06 & 5280,90 & 5,12 & 0,03 \\
\hline $\mathrm{Fc}$ & $170,05^{*}$ & $154,38^{*}$ & $24,67^{*}$ & $290,62^{*}$ & $55,31^{*}$ & $12,83^{*}$ \\
\hline $\mathrm{CV}(\%)$ & 14,42 & 14,00 & 29,09 & 8,92 & 14,62 & 25,03 \\
\hline
\end{tabular}

*Significativo a 5\%. 
Plantas com sistema radicular robusto como Panicum maximum também demonstram capacidade de rebrota, em trabalho conduzido por Melo et al. (2013) foi observada essa característica e essa foi superada com o acréscimo na dose do herbicida isoxaflutole, o que permite relacionar o controle do rebrote eficiente com o ocorrido no estádio inicial das plantas de $T$. paniculatum pelo mesmo herbicida.

O controle insuficiente observado aos 28 DAA para os herbicidas amônio glufosinato e glyphosate (1000 g e.a. ha $\left.{ }^{-1}\right)$ se justifica pela ação de contato do primeiro que atingindo apenas a parte aérea teve efeito sobre esta e não chega a porção radicular, permitindo a rebrota que é uma característica dessa planta daninha, principalmente no primeiro estádio de desenvolvimento devido ao propágulo utilizado ainda conter grande quantidade de reserva abaixo do solo e no caso do glyphosate (1000 g e.a. ha $^{-1}$ ), a recuperação das plantas ocorreu possivelmente pela dose insuficiente e baixa capacidade de translocação do herbicida. Informações semelhantes foram constatadas para capim-amargoso por Barroso et al. (2014) onde o herbicida paraquat que também possui ação dessecante permitiu a rebrota das plantas daninhas. Ocorrência de rebrota em plantas daninhas também foi relatada por Procópio et al. (2006), que ao usar o herbicida dessecante paraquat em mistura com o diuron verificaram a redução do controle. Clemente (2009) observou rebrota da espécie Cyperus rotundus nas doses de 920 e $1920 \mathrm{~g} \mathrm{ha}^{-1}$ para o herbicida glyphosate, assim como no presente trabalho a reserva do sistema radicular e propágulos das plantas foram a justificativa da ocorrência de rebrota.

Tabela 2. Efeito dos tratamentos sob o controle de T. paniculatum no estádio 1 de desenvolvimento (4-6 folhas) aos 7, 14, 21 e 28 DAA. Piracicaba - SP, 2013.

\begin{tabular}{lrrrr}
\hline \multirow{2}{*}{ Tratamentos } & \multicolumn{4}{c}{$\%$ de controle } \\
\cline { 2 - 5 } & \multicolumn{1}{c}{ 7 DAA } & 14 DAA & $21 \mathrm{DAA}$ & $28 \mathrm{DAA}$ \\
\hline 1. Amônio glufosinato & $83,33 \mathrm{a}$ & $60,00 \mathrm{~b}$ & $10,00 \mathrm{c}$ & $5,00 \mathrm{e}$ \\
2. Glyphosate $\left(1000 \mathrm{~g}\right.$ e.a. ha $\left.^{-1}\right)$ & $80,00 \mathrm{a}$ & $78,33 \mathrm{a}$ & $35,00 \mathrm{~b}$ & $20,00 \mathrm{~d}$ \\
3. Glyphosate $\left(2000 \mathrm{~g} \mathrm{e} . \mathrm{a} . \mathrm{ha}^{-1}\right)$ & $46,67 \mathrm{~b}$ & $61,66 \mathrm{~b}$ & $48,33 \mathrm{~b}$ & $68,33 \mathrm{~b}$ \\
4. Imazapic & $48,33 \mathrm{~b}$ & $63,33 \mathrm{~b}$ & $70,00 \mathrm{a}$ & $73,33 \mathrm{ab}$ \\
5. Ametryn & $50,00 \mathrm{~b}$ & $65,00 \mathrm{~b}$ & $70,00 \mathrm{a}$ & $76,67 \mathrm{ab}$ \\
6. Diuron + Hexazinone & $50,00 \mathrm{~b}$ & $65,00 \mathrm{~b}$ & $70,00 \mathrm{a}$ & $78,33 \mathrm{ab}$ \\
7. 2,4-D & $23,33 \mathrm{c}$ & $31,67 \mathrm{c}$ & $33,33 \mathrm{~b}$ & $45,00 \mathrm{c}$ \\
8. Isoxaflutole & $48,33 \mathrm{~b}$ & $68,33 \mathrm{~b}$ & $70,00 \mathrm{a}$ & $85,00 \mathrm{a}$ \\
9. Testemunha & $0,00 \mathrm{~d}$ & $0,00 \mathrm{~d}$ & $0,00 \mathrm{c}$ & $0,00 \mathrm{e}$ \\
\hline DMS & 9,684 & 9,785 & 15,408 & 12,773 \\
\hline Média & 47,78 & 54,82 & 45,56 & 50,19 \\
\hline
\end{tabular}

*Médias na coluna seguidas da mesma letra não diferem entre si, a 5\% de probabilidade pelo teste Tukey.

A capacidade de rebrota de algumas plantas daninhas é um problema para o manejo das mesmas, isso foi observado nos tratamentos com amônio glufosinato e glyphosate na menor dose. A característica de grande quantidade de água nas folhas e sistema radicular com grande capacidade de armazenamento de reservas dessa planta (Mendoza e Wood, 2013), pode ter sido a causa do comportamento de diminuição da fitointoxicação com o passar dos dias. Isso foi observado anteriormente em Bidens pilosa e
Commelina benghalensis, onde as plantas reagiram de forma semelhante (Lemos et al., 2013).

Possivelmente essa característica de rebrota se fundamenta no grande desenvolvimento do sistema radicular de $T$. paniculatum, característica essa descrita para várias espécies da família das portulacaceas (Carvalho, 2013).

No experimento 2 (Tabela 3), onde o estádio das plantas era vegetativo, porém com 
maior desenvolvimento, pode-se notar que os herbicidas com maior capacidade de absorção, por meio de raízes, caule e folhas tiveram controle mais efetivo. O glyphosate na maior dose obteve $100 \%$, ou seja, causou a morte das plantas, o diuron + hexazinone e 2,4-D também apresentaram alta eficiência, com valores acima de $90 \%$. Cabe salientar que houve a necessidade de uma dose alta de glyphosate para que valores satisfatórios de controle fossem atingidos, isso demonstra uma característica de considerável tolerância ao herbicida, já que na menor dose do mesmo não se observou bom desempenho.

Tabela 3. Efeito dos tratamentos sob o controle de $T$. paniculatum no estádio 2 de desenvolvimento (10-12 folhas) aos 7, 14, 21 e 28 DAA. Piracicaba - SP, 2013.

\begin{tabular}{lrrrr}
\hline \multirow{2}{*}{ Tratamentos } & \multicolumn{4}{c}{$\%$ de controle } \\
\cline { 2 - 5 } & \multicolumn{1}{c}{ 7 DAA } & $14 \mathrm{DAA}$ & $21 \mathrm{DAA}$ & \multicolumn{1}{c}{$28 \mathrm{DAA}$} \\
\hline 1. Amônio glufosinato & $67,50 \mathrm{a}$ & $72,50 \mathrm{~b}$ & $72,50 \mathrm{c}$ & $75,00 \mathrm{~d}$ \\
2. Glyphosate $\left(1000 \mathrm{~g} \mathrm{e} . \mathrm{a} . \mathrm{ha}^{-1}\right)$ & $25,00 \mathrm{~b}$ & $67,50 \mathrm{bc}$ & $72,50 \mathrm{c}$ & $72,50 \mathrm{~d}$ \\
3. Glyphosate $\left(2000 \mathrm{~g} \mathrm{e.a.} \mathrm{ha-} \mathrm{ha}^{-1}\right)$ & $72,50 \mathrm{a}$ & $100,00 \mathrm{a}$ & $100,00 \mathrm{a}$ & $100,00 \mathrm{a}$ \\
4. Imazapic & $13,75 \mathrm{bc}$ & $62,50 \mathrm{bcde}$ & $72,50 \mathrm{c}$ & $77,50 \mathrm{~cd}$ \\
5. Ametryn & $16,25 \mathrm{bc}$ & $51,25 \mathrm{de}$ & $85,00 \mathrm{~b}$ & $85,00 \mathrm{bc}$ \\
6. Diuron + Hexazinone & $10,00 \mathrm{~cd}$ & $47,50 \mathrm{e}$ & $88,75 \mathrm{~b}$ & $91,25 \mathrm{ab}$ \\
7. 2,4-D & $22,50 \mathrm{~b}$ & $66,25 \mathrm{bcd}$ & $90,00 \mathrm{~b}$ & $92,50 \mathrm{ab}$ \\
8. Isoxaflutole & $13,75 \mathrm{bc}$ & $55,00 \mathrm{cde}$ & $70,00 \mathrm{c}$ & $70,00 \mathrm{~d}$ \\
9. Testemunha & $0,00 \mathrm{~d}$ & $0,00 \mathrm{f}$ & $0,00 \mathrm{~d}$ & $0,00 \mathrm{e}$ \\
\hline DMS & 12,200 & 15,572 & 8,771 & 8,771 \\
\hline Média & 26,81 & 58,06 & 72,36 & 73,75 \\
\hline
\end{tabular}

*Médias na coluna seguidas da mesma letra não diferem entre si, a 5\% de probabilidade pelo teste Tukey.

Os herbicidas que obtiveram destaque na porcentagem de controle nos primeiros estádios de desenvolvimento foram favorecidos pela condição das plantas mais jovens, que são mais facilmente controladas, pois o sistema radicular ainda não apresenta estrias ou faixas de Caspary, além disso, possuem uma quantidade grande de tecido meristemático (Silva, 2006). O que favorece a atuação dos herbicidas com absorção via sistema radicular como o isoxaflutole, de absorção e translocação via simplasto como o glyphosate, com absorção tanto foliar como radicular rápida e principal efeito na região meristemática como o 2,4-D e o diuron + hexazinone (Rodrigues e Almeida, 2011).

Mesmo não havendo diferença estatística entre diuron + hexazinone e 2,4-D e esses em relação ao glyphosate na maior dose (2.000 g e.a. ha ${ }^{-1}$ ), esse é eficiente na condição de pós-emergência para $T$. paniculatum, com 10-12 folhas. Porém sua utilização em culturas não resistentes se torna inviável.
Para a associação de herbicidas se verifica eficiência maximizada, devido ao efeito sinérgico. Essa informação foi anteriormente disponibilizada em trabalho realizado com canade-açúcar, onde se verificou controle total de Ipomoea sp. com a mistura (Giancotti et al., 2014).

No caso do 2,4-D houve concordância com resultados anteriormente obtidos na dessecação de mamona, onde o mesmo proporcionou grande perda de água pelas plantas, ocorrendo sua dessecação completa de forma eficiente (Foloni et al., 2011).

Resultados semelhantes foram relatados em um trabalho utilizando glyphosate em doses crescentes em Commelina benghalensis e Cyperus rotundus, após 30 dias os tratamentos de maior dose haviam atingido valores significativamente altos de controle, considerando que essas espécies são agressivas, se pode inferir que o produto é efetivo nas maiores doses e permite a recomendação de seu 
posicionamento nessas situações (Santos Júnior et al., 2013).

Para o estádio reprodutivo das plantas (experimento 3), a maioria dos tratamentos demonstrou baixa eficiência (Tabela 4), tendo os valores ficado próximos aos $30 \%$, isso provavelmente devido ao aumento da tolerância das plantas conforme seu desenvolvimento vegetativo. Situação essa observada para a espécie Commelina sp. que a medida em que se aumentaram as doses de glyphosate aplicadas, essas tenderam a se tornar menos efetivas, demonstrando certa tolerância, que foi diretamente relacionada com a fase mais avançada de desenvolvimento das plantas (Dias et al., 2013). Semelhante resultado foi observado por Cesarin et al. (2013) para a planta daninha Sisyrinchium micranthum Cav. Em estádio reprodutivo, onde os herbicidas testados também tiveram redução de controle conforme passou o tempo após a aplicação, esse resultado foi atribuído as características da planta e ao estádio no momento da aplicação.

Tabela 4. Efeito dos tratamentos sob o controle de T. paniculatum no estádio 3 de desenvolvimento (10-12 folhas e inflorescência) aos 7, 14, 21 e 28 DAA. Piracicaba - SP, 2013.

\begin{tabular}{|c|c|c|c|c|}
\hline \multirow{2}{*}{ Tratamentos } & \multicolumn{4}{|c|}{$\%$ de controle } \\
\hline & 7 DAA & $14 \mathrm{DAA}$ & $21 \mathrm{DAA}$ & $28 \mathrm{DAA}$ \\
\hline 1. Amônio glufosinato & $95,00 \mathrm{a}$ & $92,50 \mathrm{a}$ & $90,00 \mathrm{a}$ & $88,75 \mathrm{~b}$ \\
\hline 2. Glyphosate $(1000$ g e.a. ha-1) & $22,50 \mathrm{~b}$ & $27,50 \mathrm{c}$ & $27,50 \mathrm{~cd}$ & $27,50 \mathrm{~cd}$ \\
\hline 3. Glyphosate $\left(2000\right.$ g e.a. ha $\left.^{-1}\right)$ & $25,00 \mathrm{~b}$ & $47,50 \mathrm{~b}$ & $71,25 \mathrm{ab}$ & $93,75 \mathrm{ab}$ \\
\hline 4. Imazapic & $25,00 \mathrm{~b}$ & $25,00 \mathrm{c}$ & $33,75 \mathrm{c}$ & $33,75 \mathrm{c}$ \\
\hline 5. Ametryn & $18,75 \mathrm{~b}$ & $18,75 \mathrm{c}$ & $22,50 \mathrm{~cd}$ & $22,50 \mathrm{~d}$ \\
\hline 6. Diuron + Hexazinone & $16,25 \mathrm{~b}$ & $17,50 \mathrm{c}$ & $41,25 \mathrm{bc}$ & $30,00 \mathrm{~cd}$ \\
\hline 7. $2,4-\mathrm{D}$ & $83,75 \mathrm{a}$ & $95,00 \mathrm{a}$ & $100,00 \mathrm{a}$ & $100,00 \mathrm{a}$ \\
\hline 8. Isoxaflutole & $22,50 \mathrm{~b}$ & $23,75 \mathrm{c}$ & $30,00 \mathrm{~cd}$ & $33,75 \mathrm{c}$ \\
\hline 9. Testemunha & $0,00 \mathrm{c}$ & $0,00 \mathrm{~d}$ & $0,00 \mathrm{~d}$ & $0,00 \mathrm{e}$ \\
\hline DMS & 11,894 & 12,996 & 32,339 & 10,248 \\
\hline Média & 34,31 & 38,61 & 46,25 & 47,78 \\
\hline
\end{tabular}

*Médias na coluna seguidas da mesma letra não diferem entre si, a 5\% de probabilidade pelo teste Tukey.

O herbicida que apresentou melhor nível de controle foi o 2,4-D, seguido por glyphosate na maior dose e amônio glufosinato que tiveram eficiência nessa fase das plantas, o glyphosate por demonstrar novamente efeito crescente durante os dias após aplicação e ação sistêmica e o amônio glufosinato por proporcionar com seu efeito de contato injurias não mais revertidas pelas plantas de $T$. paniculatum.

Porém o destaque está no 2,4-D que controlou totalmente as plantas, mesmo não diferindo estatisticamente do tratamento com glyphosate em maior dose, nesse estádio o efeito de mimetização da auxina produzido por ele ocasionou rachaduras nos órgãos das plantas, assim como epinastia e hipertrofia radicular que fizeram com que nessa fase o 2,4-D se destacasse e obtivesse controle total logo aos 21 DAA.

Em trabalho conduzido com esse herbicida sozinho e associado ao glyphosate as informações geradas demonstraram que 0 mesmo é efetivo tanto sozinho como associado após os 33 dias da aplicação, e que em plantas maiores e em fase de desenvolvimento avançado o controle foi superior aos $90 \%$, o que sugere sua indicação e utilização (Santos et al., 2002).

Destaca-se também que a maior dose de glyphosate $2.000 \mathrm{~g}$ e.a ha ${ }^{-1}$ que foi necessária para o controle total é relativamente alta para plantas do porte de T. paniculatum, gerando com essa observação uma indicação de tolerância da mesma ao herbicida. 
Para as variáveis massa fresca e seca (Tabela 5), não se pode verificar um padrão na redução de massa, porém os maiores níveis de controle tiveram relação direta com a diminuição de massa das plantas de $T$. paniculatum, dentro dos três experimentos, ou seja, confirmando as respostas obtidas para cada fase das plantas daninhas.

Tabela 5. Efeito dos tratamentos sob a massa fresca (MF) e seca (MS) em gramas das plantas de T. paniculatum nos estádios 1, 2 e 3, aos 28 DAA. Piracicaba - SP, 2013.

\begin{tabular}{lrrrrrr}
\hline \multirow{2}{*}{ Tratamentos } & \multicolumn{2}{c}{ Estádio 1 } & \multicolumn{2}{c}{ Estádio 2 } & \multicolumn{2}{c}{ Estádio 3 } \\
\cline { 2 - 7 } & MF $(\mathrm{g})$ & MS $(\mathrm{g})$ & MF $(\mathrm{g})$ & $\mathrm{MS}(\mathrm{g})$ & MF $(\mathrm{g})$ & \multicolumn{1}{c}{ MS $(\mathrm{g})$} \\
\hline 1. Amônio glufosinato & $5,990 \mathrm{~b}$ & $0,430 \mathrm{~b}$ & $1,840 \mathrm{~cd}$ & $0,155 \mathrm{~d}$ & $0,505 \mathrm{c}$ & $0,223 \mathrm{~b}$ \\
2. Glyphosate $\left(1000 \mathrm{~g}\right.$ e.a. $\left.\mathrm{ha}^{-1}\right)$ & $5,376 \mathrm{~b}$ & $0,363 \mathrm{~b}$ & $1,990 \mathrm{~cd}$ & $0,213 \mathrm{~cd}$ & $2,845 \mathrm{ab}$ & $0,308 \mathrm{ab}$ \\
3. Glyphosate $\left(2000 \mathrm{~g} \mathrm{e} . \mathrm{a} . \mathrm{ha}^{-1}\right)$ & $1,450 \mathrm{~d}$ & $0,187 \mathrm{c}$ & $0,265 \mathrm{~d}$ & $0,175 \mathrm{~d}$ & $0,698 \mathrm{c}$ & $0,188 \mathrm{~b}$ \\
4. Imazapic & $1,416 \mathrm{~d}$ & $0,177 \mathrm{c}$ & $5,623 \mathrm{a}$ & $0,530 \mathrm{a}$ & $2,937 \mathrm{ab}$ & $0,273 \mathrm{ab}$ \\
5. Ametryn & $2,133 \mathrm{c}$ & $0,157 \mathrm{c}$ & $2,200 \mathrm{c}$ & $0,485 \mathrm{ab}$ & $2,677 \mathrm{~b}$ & $0,273 \mathrm{ab}$ \\
6. Diuron + Hexazinone & $1,296 \mathrm{~d}$ & $0,047 \mathrm{c}$ & $1,735 \mathrm{~cd}$ & $0,360 \mathrm{abcd}$ & $2,425 \mathrm{~b}$ & $0,240 \mathrm{~b}$ \\
7. 2,4-D & $1,596 \mathrm{~cd}$ & $0,423 \mathrm{~b}$ & $2,503 \mathrm{bc}$ & $0,240 \mathrm{~cd}$ & $0,700 \mathrm{c}$ & $0,138 \mathrm{bc}$ \\
8. Isoxaflutole & $1,623 \mathrm{~cd}$ & $0,153 \mathrm{c}$ & $3,433 \mathrm{bc}$ & $0,280 \mathrm{bcd}$ & $2,438 \mathrm{~b}$ & $0,213 \mathrm{~b}$ \\
9. Testemunha & $8,000 \mathrm{a}$ & $1,000 \mathrm{a}$ & $4,238 \mathrm{ab}$ & $0,435 \mathrm{abc}$ & $3,500 \mathrm{a}$ & $0,350 \mathrm{a}$ \\
\hline DMS & 0,679 & 0,141 & 1,815 & 0,233 & 0,731 & 0,124 \\
\hline Média & 3,209 & 0,326 & 2,647 & 0,319 & 2,081 & 0,245 \\
\hline
\end{tabular}

*Médias na coluna seguidas da mesma letra não diferem entre si, a 5\% de probabilidade pelo teste Tukey.

Comportamento esse já esperado e coerente com o que se busca na utilização do método químico de controle, pois essa redução de massa significa efetividade de controle, diminuição da taxa metabólica e em vários casos morte da planta daninha. Concordando com o que foi anteriormente exposto, Opeña et al. (2014) verificaram redução massa seca de plantas daninhas, dentre elas Portulaca oleraceae devido ao manejo e utilização de herbicidas nas culturas do trigo e do milho.

De forma geral o trabalho demonstrou a variação na resposta de controle químico das plantas de maria gorda nos seus distintos estádios de desenvolvimento, isso também foi observado para a massa fresca e seca, e ficou demonstrada capacidade de tolerância dessa espécie a alguns tratamentos, em especial ao glyphosate na menor dosagem em estádios específicos de desenvolvimento.

Portanto são necessárias mais abordagens dessa espécie no que tange seu manejo como planta daninha, mais herbicidas e doses, assim como opções na tecnologia de aplicação que auxiliem a geração de mais informações pertinentes ao método químico, considerando-se que a escolha e posicionamento do herbicida são fundamentais para isso, o que se intensifica nos casos de plantas agressivas como $T$. paniculatum. Até mesmo o teste de outros métodos de controle é desejável, já que se trata de uma espécie ainda pouco explorada no âmbito da matologia.

\section{Conclusões}

O uso de herbicidas se mostrou eficiente na minoria dos arranjos testados para o controle de T. paniculatum.

Com destaque para isoxaflutole no estádio 1 devido a forma de absorção via sistema radicular que inibiu a rebrota, glyphosate na maior dose, 2,4-D e diuron + hexazinone no estádio 2. Glyphosate na maior dose e 2,4-D no estádio 3.

O estádio de desenvolvimento da planta pode influenciar a eficácia de controle dos tratamentos.

\section{Referências}

BARROSO, A. et al.Accase and glyphosate diferent formulations herbicides association 
interactions on sourgrass control. Planta Daninha, v.32, n.3, p.619-627, 2014.

BORCHARTT, L. et al. Períodos de interferência de plantas daninhas na cultura do feijoeiro-comum (Phaseolus vulgaris L.). Revista Ciência Agronômica, v.42, n.3, p.725734, 2011.

CARVALHO, L.B. Classificações. In: CARVAlHO, L.B. (Ed.) Plantas daninhas. Lages: FAESB, 2013. cap. 5, p.35-48.

CLEMENTE, T.B. Efeitos da aplicação de ácido giberélico na susceptibilidade de Cyperus rotundus L. ao glifosato. 2009. $44 \mathrm{f}$. Dissertação (Mestrado em Ciencias Biológicas) - Universidade do Estado de São Paulo, Botucatu, 2009.

CONCENÇO, G. et al. Occurrence of weed species in Jatropha curcas intercropping systems. Planta Daninha, v.32, n.2, p.327-334, 2014.

DIAS, A.C.R.; CARVALHO, S.J.P.; CHRISTOFFOLETI, P.J. Fenologia da trapoeraba como indicador para tolerância ao herbicida glyphosate. Planta Daninha, v.31, n.1, p.185-191, 2013.

EMBRAPA. Sistema de classificação de solos. Rio de Janeiro, RJ: 2006.

FOLONI, J.S.S. et al. Dessecação química em pré-colheita da mamona. Revista Ceres, v.58, n.5, p.665-669, 2011.

FREITAS, F. et al. Interferência de plantas daninhas na cultura do feijão-caupi. Planta Daninha, v.27, n.2, p.241-247, 2009.

GIANCOTTI, P.R.F. et al. Chemical control of morning glory as a function of water restriction levels. Planta Daninha, v.32, n.2, p.345-353, 2014.

GOMES, G. et al. Weed community assessment in the banana culture. Planta Daninha, v.28, n.1, p.61-68, 2010.

KUVA, M. et al. Weed seedbank and its correlation with the established flora in no-burn sugarcane area. Planta Daninha, v.26, n.4, p.735-744, 2008.

LEMOS, J. et al. Management of Bidens pilosa and Commelina benghalensis in organic corn cultivation under no-tillage. Planta Daninha, v.31, n.2, p.351-357, 2013.

MACHADO FILHO, H. et al. Composition and similarity of flora associated with anthropogenic sites in the city of João Pessoa-Paraíba. Planta Daninha, v.33, n.1, p.57-66, 2015.

MACIEL, C.D.G.; SOUZA, J.I.; HAMA, J.T. Levantamento fitossociológico de plantas daninhas em jardins residenciais com grama esmeralda em ourinhos-sp. Global Science and Technology, v.3, n.2, p.39-48, 2010.

MARCHIORI JR., O. et al. Efeito residual de isoxaflutole após diferentes períodos de seca. Planta Daninha, v.23, n.3, p.491-499, 2005.

MOURA FILHO, E.R.; MACEDO, L.P.M.; SILVA, A.R.S. Levantamento fitossociológico de plantas daninhas em cultivo de banana irrigada. HOLOS, v.2, s/n, p.92-97, 2015.

NASCIMENTO, P.G.M.L. et al. Levantamento fitossociológico das comunidades infestantes em diferentes sistemas de plantio de milho em Mossoró-RN. Agropecuária Científica no Semiárido, v.7, n.3, p.1-9, 2012.

OLIVEIRA, A.; FREITAS, S. Levantamento fitossociológico de plantas daninhas em áreas de produção de cana-de-açúcar. Planta Daninha, v.26, n.1, p.33-46, 2008.

OPEÑA, J.L. et al. Weed population dynamics, herbicide efficacies, and crop performance in a sprinkler-irrigated maize-rice cropping system. Field Crops Research, v.167, s/n, p.119-130, 2014.

PROCÓPIO, S.O. et al. Efeitos de dessecantes no controle de plantas daninhas na cultura da soja. Planta Daninha, v.24, número especial, p.193-197, 2006.

RODRIGUES, B.N.; ALMEIDA, F.S. Guia de herbicidas. $6^{\text {a }}$. Londrina, PR: 2011. 697p. 
SANTOS JÚNIOR, A. et al. Manejo de tiririca e trapoeraba com glyphosate em ambientes sombreados. Planta Daninha, v.31, n.1, p.213221, 2013.

SANTOS, I.C.D. et al. Eficiência do 2, 4-D aplicado isoladamente e em mistura com glyphosate no controle da trapoeraba. Planta Daninha, v.20, n.2, p.299-309, 2002.

SILVA, A.A. et al. Biologia de plantas daninhas. In: SILVA, A. A.; SILVA, J. F. Tópicos em manejo de plantas daninhas. Viçosa, MG: Universidade Federal de Viçosa, 2006. cap 1, 318 p.

SOARES, I. et al. Interferência das plantas daninhas sobre a produtividade e qualidade de cenoura. Planta Daninha, v.28, n.2, p.247-254, 2010.

SOCIEDADE BRASILEIRA DA CIÊNCIA DAS PLANTAS DANINHAS - SBCPD. Procedimentos para instalação, avaliação e análise de experimentos com herbicidas. SBCPD, Londrina: 42p. 1995. 